Artículo científico

(Original paper)

\title{
REVISIÓN GENÉRICA DE FULCIDACINI JAKOBSON, 1924 (CHRYSOMELIDAE, CRYPTOCEPHALINAE) PARA MÉXICO
}

\author{
LILIANA HERNÁNDEZ SOSA ${ }^{1 *}$, JESÚS ROMERO NÁPOLES ${ }^{1}$, JORGE MANUEL VALDEZ \\ CARRASCO ${ }^{1}$, JOSÉ LUIS CARRILLO SÁNCHEZ ${ }^{1}$, SAMUEL RAMÍREZ ALARCÓN ${ }^{2}$, MARÍA MAGDALENA \\ ORDÓÑEZ RESÉNDIZ ${ }^{3}$, SANTIAGO NIÑO MALDONADO ${ }^{4}$
}

\author{
${ }^{1}$ Especialidad de Entomología y Acarología. Instituto de Fitosanidad. Colegio de Postgraduados. C.P. 56230. \\ Montecillo, Estado de México.<neochlamisus@gmail.com>; <jnapoles@colpos.mx>; <jvaldez@colpos.mx>; \\ <josecarr@colpos.mx> \\ ${ }^{2}$ Parasitología Agrícola Universidad Autónoma de Chapingo, Carretera México - Texcoco, Texcoco, Estado de México, \\ Km. 38.5, C. P. 35230. <ramirezsamuel@ hotmail.com> \\ ${ }^{3}$ Colección Coleopterológica, Museo de Zoología, Facultad de Estudios Superiores Zaragoza, Universidad Nacional \\ Autónoma de México. Av. Guelatao 66, Ejército de Oriente, Iztapalapa, CDMX., México, C. P. 09230. <mor@unam.mx> \\ ${ }^{4}$ Universidad Autónoma de Tamaulipas, Facultad de Ingeniería y Ciencias, Centro Universitario Victoria, Cd. Victoria, \\ Tamaulipas, México. C. P. 87149. <coliopteranino@hotmail.com> \\ *Autor de correspondencia: <neochlamisus@gmail.com>
}

Recibido: 05/10/2017; aceptado: 09/10/2018; publicado en línea: 29/03/2019

Editor responsable: Pedro Reyes-Castillo†; Magdalena Cruz Rosales

\section{Hernández-Sosa, L., Romero-Nápoles, J., Valdez-Carrasco, J. M., Carrillo-Sánchez, J. L., Ramírez- Alarcón, S., Ordóñez-Reséndiz, M. M., Niño-Maldonado, S. (2019) Revisión genérica de Fulcidacini Jakobson, 1924 (Chrysomelidae, Cryptocephalinae) para México. Acta Zoológica Mexicana (nueva serie), 35, 1-16. https://doi.org/10.21829/azm.2019.3502198}

RESUMEN. Los fulcidacinos son insectos fitófagos que se caracterizan por la presencia de tubérculos pronotales y elitrales bien desarrollados, lo que les permite confundirse con pequeños terrones o incluso con excretas de otros insectos. A nivel mundial la tribu Fulcidacini cuenta con 500 especies, incluidas en 11 géneros. De acuerdo con los listados de especies, para México se estimaban entre 54 y 74 especies, mientras que en este trabajo se registran 76 especies. Sin embargo, actualmente son escasas las claves taxonómicas para la identificación de especies mexicanas, por ello con el propósito de contribuir al conocimiento de la tribu en México, en este trabajo se presenta la revisión taxonómica de los géneros de Fulcidacini registrados para el país. Se proporciona una clave dicotómica para su identificación y se ilustran especies representativas. También se incluye la lista de especies mexicanas de cada género, con datos de distribución estatal. Exema punctatipes es transferida al género Aulacochlamys por las carinas presentes en el pronoto; una característica particular del género.

Palabras clave: Taxonomía; géneros; clave; distribución

\begin{abstract}
The fulcidacinos are phytophagous insects characterized by the presence of well-developed pronotal and elytral tubercles, which lead them to be confused with small clods or even excrement from other insects. Worldwide, the tribe Fulcidacini has 500 species, included in 11 genera. According to the species lists, were estimated that there are 54 to 74 species for Mexico, 76 species are recorded in this work. However, the taxonomic keys for identification of Mexican species are scarce; thus, with the purpose to contribute to the knowledge of the tribe in Mexico, taxonomic revision of the genera of Fulcidacini


registered for the country is presented in this work. A dichotomous key is provided for identification and representative species are illustrated. Also included is a list of the Mexican species of each genus, with data on state distribution. Exema punctatipes is transferred to the genus Aulacochlamys due the carinae present in the pronotum; a particular feature of the genus.

Key words: Taxonomy; genera; key; distribution

\section{INTRODUCCIÓN}

La tribu Fulcidacini y sus tribus hermanas Clytrini y Cryptocephalini forman parte del grupo monofilético Camptosomata, es decir, las especies que constituyen a este grupo descienden de un ancestro común. Dichas tribus comparten un carácter preimaginal que consiste en la construcción de una caja protectora. Esta caja la comienza a construir la hembra después de adherir el huevo a la planta, mismo que cubre por completo de excreta empleando sus patas posteriores y pigidio; después, al emerger la larva carga su caja protectora adhiriendo más desecho conforme va creciendo, cuando su estado larvario está por concluir sella su caja y se prepara para pupar (Reid, 1991; Brown \& Funk, 2005).

Las características morfológicas que separan a la tribu Fulcidacini de Clytrini y Cryptocephalini, son: la presencia de tubérculos (elevaciones o surcos irregulares) en los élitros y el pronoto, en el mesotórax y metatórax se presentan depresiones pronunciadas que sirven para ocultar por completo sus patas, así mismo las antenas se ocultan en surcos ubicados en la zona lateral del proesterno. Con las características mencionadas, los insectos generalmente parecen pequeños terrones o excretas de otros insectos (Fig. 5) (Monrós, 1951a; Chamorro-Lacayo \& Konstantinov, 2009).

La tribu Fulcidacini antes se conocía con el nombre de Chlamisinae o como la tribu Chlamisini al considerar como género tipo a Chlamys Knoch, 1801, nombre que resultó inválido de acuerdo con el Código Internacional de Nomenclatura Zoológica, por ser homónimo de un género de molusco bivalvo. Fue en 1924 cuando Jakobson consideró como género tipo a Fulcidax Voet, 1806 por ser el siguiente nombre disponible más antiguo (Karren, 1972). En el trascurso de los años la tribu había variado en sus diferentes nombres de acuerdo con la postura de cada autor, fue a partir de la clasificación de Bezděk (2010) y Bouchard et al. (2011) que el nombre más aceptado es Fulcidacini Jakobson, 1924, por lo cual, el criterio de estos autores se tomó en cuenta para este trabajo.

Como la mayoría de los crisomélidos, los fulcidacinos son insectos fitófagos, a nivel de especie se consideran monófagos y de manera general pueden alimentarse de diversas plantas (Jolivet et al., 1988). Flowers \& Hanson (2003) mencionan que su función en el ecosistema es la reincorporación de materia orgánica, controlando así el crecimiento de las poblaciones de plantas, es por ello que algunas especies pueden ser aprovechadas como agentes para el biocontrol de malezas, tal es el caso de Chlamisus mimosae Karren, una especie que se ha empleado en Australia para controlar el crecimiento de la maleza Mimosa pigra L. (Waterhouse, 1994; Harley et al., 1995; Ostermeyer \& Grace, 2006). También se han reportado especies de importancia agrícola, entre ellas se encuentran: Neochlamisus platini (Brown, 1952) que infestó árboles ornamentales de Platanus occidentalis L. en Estados Unidos (Hyche, 1996), Chlamisus cribripennis (LeConte) se registró defoliando arándano en Canadá (Wood, 1966) y Fulcidax coelestina (Lacordaire, 1848) como defoliador de Malpighia glabra L. en Brasil (Braga et al., 1999).

En el mundo, se estiman 500 especies incluidas en 11 géneros: Chlamisus Rafinesque, Fulcidax Voet, Carcinobaena Lacordaire, Exema Lacordaire, Hymetes Lacordaire, Pseudochlamys Lacordaire, Diplacaspis Jakobson, Melittochlamys Monrós, Aulacochlamys Monrós, Neochlamisus Karren y Kakita Chamorro-Lacayo \& Konstantinov (Chamorro-Lacayo \& Konstantinov, 2009). Ordóñez-Reséndiz (2014) registró para México seis géneros y 74 especies en el catálogo de autoridades de Chrysomelidae. Si bien 
existen algunas claves generales para la identificación genérica, como las de Lacordaire (1848), Monrós (1951a), Karren (1972) y Chamorro-Lacayo y Konstantinov (2009), para la identificación a nivel específico de México sólo existe la revisión de Karren (1972) que únicamente considera especies de Norteamérica y del norte del país; por tal razón en este estudio se realizó una revisión de las especies reportadas para México, basado en la información de ejemplares resguardados en Colecciones Entomológicas del país y en la información derivada de la revisión de literatura, obteniendo así una clave para los géneros mexicanos y un listado de las especies para cada uno de ellos.

\section{MATERIALES Y MÉTODOS}

Obtención del material entomológico. Se revisaron las colecciones de insectos de las siguientes instituciones de México: Colección de Insectos del Colegio de Postgraduados, Montecillo, Estado de México (CEAM); Colección de Insectos del Colegio de Postgraduados, Tabasco (COLPOS-T); Universidad Autónoma de Chapingo (UACh); Colección Nacional de Insectos del Instituto de Biología UNAM (CNIN); Colección Coleopterológica de la Facultad de Estudios Superiores Zaragoza UNAM (CCFES-Z); Escuela Nacional de Ciencias Biológicas del Instituto Politécnico Nacional (ENCB); Universidad Autónoma de Tamaulipas (UAT); El Colegio de la Frontera Sur, San Cristóbal de las Casas, Entomología (CEFS). El material se solicitó en préstamo para su posterior identificación en el laboratorio de Taxonomía y Sistemática del Colegio de Postgraduados, Campus Montecillo.

Identificación taxonómica. Se utilizaron las claves taxonómicas de Monrós (1951a, b), Karren (1966, 1972), Chamorro-Lacayo y Konstantinov (2009) y literatura descriptiva de géneros y especies de Fulcidacini (Lacordaire, 1848; Jacoby, 1889). Para la determinación de las especies se requirió de la extracción de las genitalias del macho y la hembra a través de la hidratación de los ejemplares en agua a $80^{\circ} \mathrm{C}$ durante 15 minutos. Bajo un estereomicroscopio, el pigidio fue abierto para separar la genitalia, misma que se maceró durante diez minutos en una solución de $\mathrm{KOH}$ al $10 \%$ a $90^{\circ} \mathrm{C}$, luego el $\mathrm{KOH}$ fue neutralizado con ácido acético al 1\%. Finalmente, se lavó con agua destilada y el genital se almacenó en un microvial con glicerina.

Se elaboró una clave taxonómica para los géneros presentes en México considerando los criterios de las claves ya existentes de Chamorro-Lacayo y Konstantinov (2009), Karren (1972) y Monrós (1951a) y los caracteres observados con claridad durante la revisión del material. Se tomaron fotografías de los insectos en un estereomicroscopio Zeiss SteREO V20 y las imágenes se capturaron con el software Darktable 2.2.5. Las fotografías que se muestran en las láminas fueron seleccionadas para la observación de los caracteres que se citan en la clave. Se enlistan las especies mexicanas para cada género en orden alfabético y en algunas especies se actualiza la distribución que se reportaba en la literatura hasta antes de este trabajo, éstas se señalan con un asterisco (*), en el caso de las especies que fueron revisadas se señala en que colección se encuentran depositadas.

\section{RESULTADOS}

Se revisaron 341 ejemplares de las ocho colecciones consultadas, de este material se obtuvieron los siguientes seis géneros ya registrados anteriormente para México: Chlamisus, Diplacaspis, Exema, Fulcidax, Melittochlamys, Neochlamisus y se transfiere una especie del género Exema al género Aulacochlamys, por presentar seis carinas distribuidas en el pronoto, además se verificaron y enriquecieron las distribuciones de 17 especies (señaladas con un asterisco) en los géneros: Chlamisus, Exema, Fulcidax Melittochlamys y Neochlamisus.

Las genitalias fueron útiles para identificar machos y hembras, se observó que como en la mayoría de los insectos, el macho es de menor tamaño que la hembra. En el caso de los machos, los edeagos 
mostraron diferentes formas entre las especies. Sin embargo, para el caso de los géneros solo existen diferencias representativas en Neochlamisus, por presentar una guía eyaculatoria asimétrica con características semejantes entre las especies que la constituyen.

\section{Tribu Fulcidacini Jakobson, 1924}

Son insectos pequeños en su mayoría $(2.1-12.0 \mathrm{~mm})$, de colores variados, brillantes, metálicos o mate, predominando el café, amarillo y negro, pueden presentar más de un color a lo largo del cuerpo; en general el cuerpo es muy esclerosado. Cabeza plana casi oculta bajo el pronoto, con ojos grandes muy escotados; piezas bucales con mandíbulas fuertes, el labro es corto y protege a los palpos maxilares. En vista dorsal los élitros y el pronoto con tubérculos reducidos o bien desarrollados; la superficie elitral y pronotal con puntos variados, glabra o pubescente. Escutelo pequeño con forma de trapecio, con la base menos ancha que el ápice; sutura elitral dentada hasta la mitad o completa, alas metatorácicas bien desarrolladas. Pigidio de superficie lisa, punteada, vermiculada, estriada, rugosa, con o sin carinas. Proesterno evidente, con surcos laterales donde se ocultan las antenas que frecuentemente son de color amarillo, café o negro. Debajo de las coxas existen depresiones dirigidas al proesterno, que sirven para ocultar cada pata (Fig. 1). El conjunto de características mencionadas permite que los fulcidacinos se mimeticen con excretas de otros insectos.

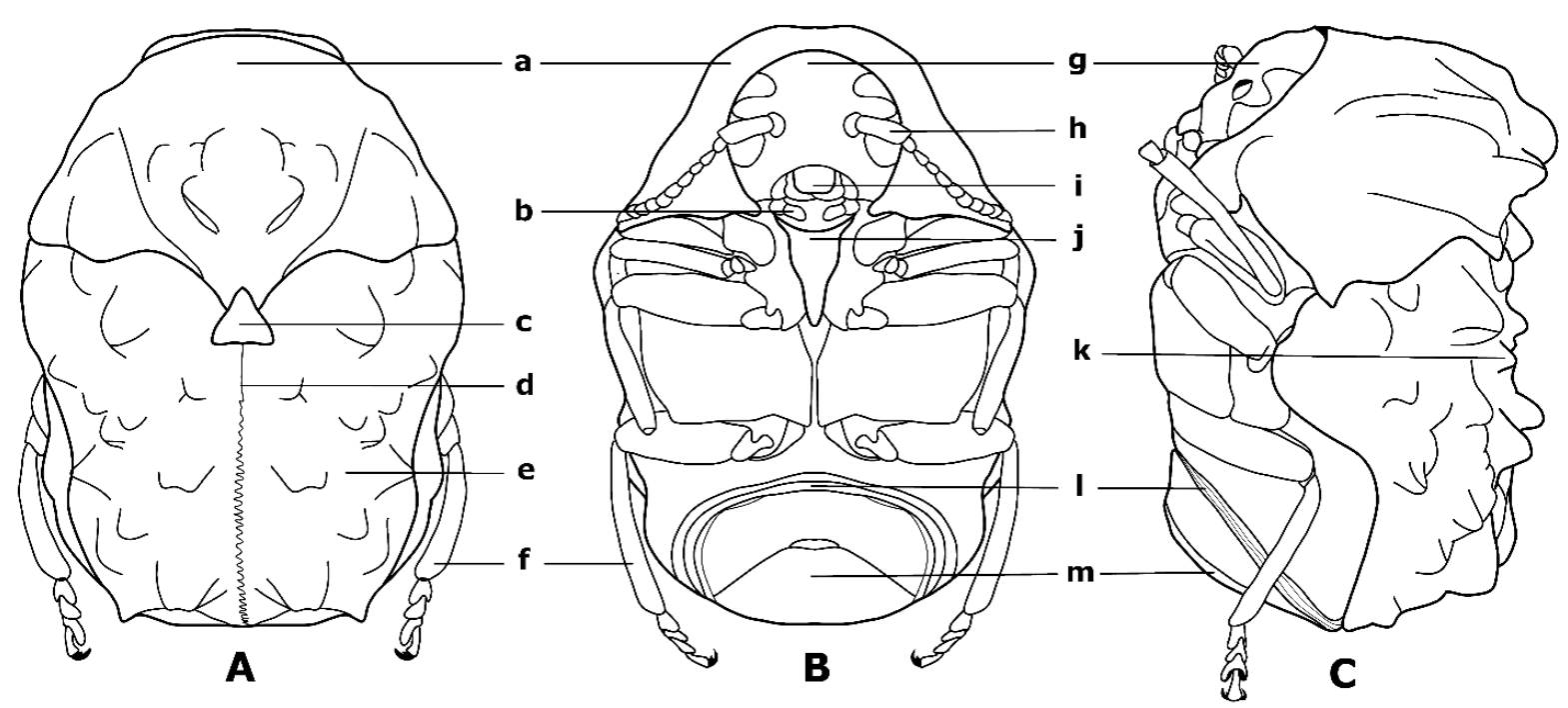

Figura 1. Morfología de Fulcidacini: A. Vista dorsal; B. Vista ventral; C. Vista dorsal; a. pronoto; b. palpo maxilar; c. escutelo; d. sutura elitral; e. élitro; f. patas; g. cabeza; h. antena; i. labro; j. proesterno; k. tubérculo o giba; 1. esternitos; m. pigidio.

\section{Clave para los géneros de Fulcidacini de México}

1. Proesterno casi rectangular entre mesocoxas, con margen posterior un poco más estrecho que el margen anterior (Fig. 7B); tubérculos pronotales y elitrales reducidos (Fig. 7A)..... Melittochlamys Monrós, 1951 $1^{\prime}$. Proesterno de forma variable, triangular o angular, nunca rectangular entre mesocoxas, con margen posterior más estrecho que el margen anterior; tubérculos pronotales y elitrales bien desarrollados (excepto algunos Chlamisus) ........................................................................... 2

2(1'). Cabeza con un surco longitudinal en el vértex; uñas tarsales simples; de color metálico brillante; pronoto y élitros con tubérculos pronunciados (Figs. 6A, 6B). 
2'. Cabeza con surco indistinto en el vértex; uñas tarsales apendiculadas (excepto algunos Exema); pronoto y élitros con tubérculos menos pronunciados.

3(2’). Proesterno cóncavo en la parte media, ápice convexo (Fig. 4B); metaescutelo expuesto (Fig. 4A)

Diplacaspis Jakobson, 1924

3'. Proesterno convexo, aserrado o sólo un poco cóncavo en la parte media; metaescutelo no expuesto

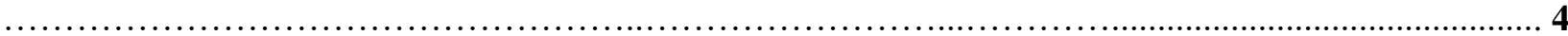

4(3'). Pronoto con 6 carinas distintivas, pequeñas, afiladas y longitudinales que convergen cerca de la base en la parte media (Fig. 2); de tamaño pequeño; por lo general de color negro............................................................... Aulacochlamys Monrós, 1951 $4^{\prime}$. Pronoto nunca con 6 carinas longitudinales que convergen medialmente cerca del margen posterior.

5(4`). Guía eyaculatoria de la genitalia del macho asimétrica, con vaina; élitro con agrupación de puntos de apariencia aterciopelada (Fig. 8A) Neochlamisus Karren, 1972 5'. Guía eyaculatoria de la genitalia del macho simétrica, sin vaina; élitros sin agrupación de puntos de apariencia aterciopelada.

6(5`). Machos con espinas o espínulas en el primer ventrito (Fig. 5B); casi siempre el quinto antenómero menor que el sexto.

Exema Lacordaire, 1848 $6^{\prime}$. Machos sin espinas o espínulas en el primer ventrito; el quinto antenómero casi tan grande como el sexto (Fig. 3B). Chlamisus Rafinesque, 1815

\section{Género Aulacochlamys Monrós, 1951}

El género fue creado por Monrós (1951a) cuando encontró cinco nuevas especies del género Exema de Argentina, caracterizadas por la presencia de seis carinas pronotales, característica que utilizo años atrás Lacordaire (1848) para crear la segunda división del género Exema. Monrós (1951a) transfirió a $A$. costicollis (Lacordaire, 1848) para describir al género y describió a A. recticarinata, A. pygidialis, A. radiata, A. minuta y A. ultima como nuevas especies del género. Las especies de Aulacochlamys en su mayoría son pequeñas, de color negro brillante y se caracteriza por la presencia de seis carinas afiladas pronotales que se extienden como abanico hacia el ápice y convergen muy cerca de la parte media de la base, pueden estar completas o cortas, rectas o sinuosas. Escutelo muy pequeño pero visible a excepción de A. octocarinata Jacoby, 1889. Élitros generalmente con cuatro carinas de distribución semejante entre las especies, pero con distinto tamaño y grosor; la superficie entre las carinas puede presentar puntos simples, profundos y/o adornados con lineolas radiadas; patas delgadas, negras o amarillas. En la mayoría de las especies en el pigidio existen tres carinas de igual o diferente tamaño, la superficie puede ser lisa, vermiculada o rugosa; por lo general el proesterno es cóncavo en la parte media, como en Diplacaspis, pero el metaescutelo nunca es visible. Por su tamaño pueden confundirse con especies del género Exema o Chlamisus; sin embargo, en ninguna existen las seis carinas pronotales que definen al grupo. Abdomen sin espinas o espínulas en el primer segmento; edeago y espermateca semejante a Chlamisus.

Más de la mitad de las especies reportadas para el mundo se encuentran en Sudamérica. Exema punctatipes (Lacordaire, 1848) registrada en México por Lacordaire (1848), es una especie con carinas pronotales, por lo que en este trabajo se transfiere al género Aulacochlamys acorde a los criterios de Monrós (1951a). 


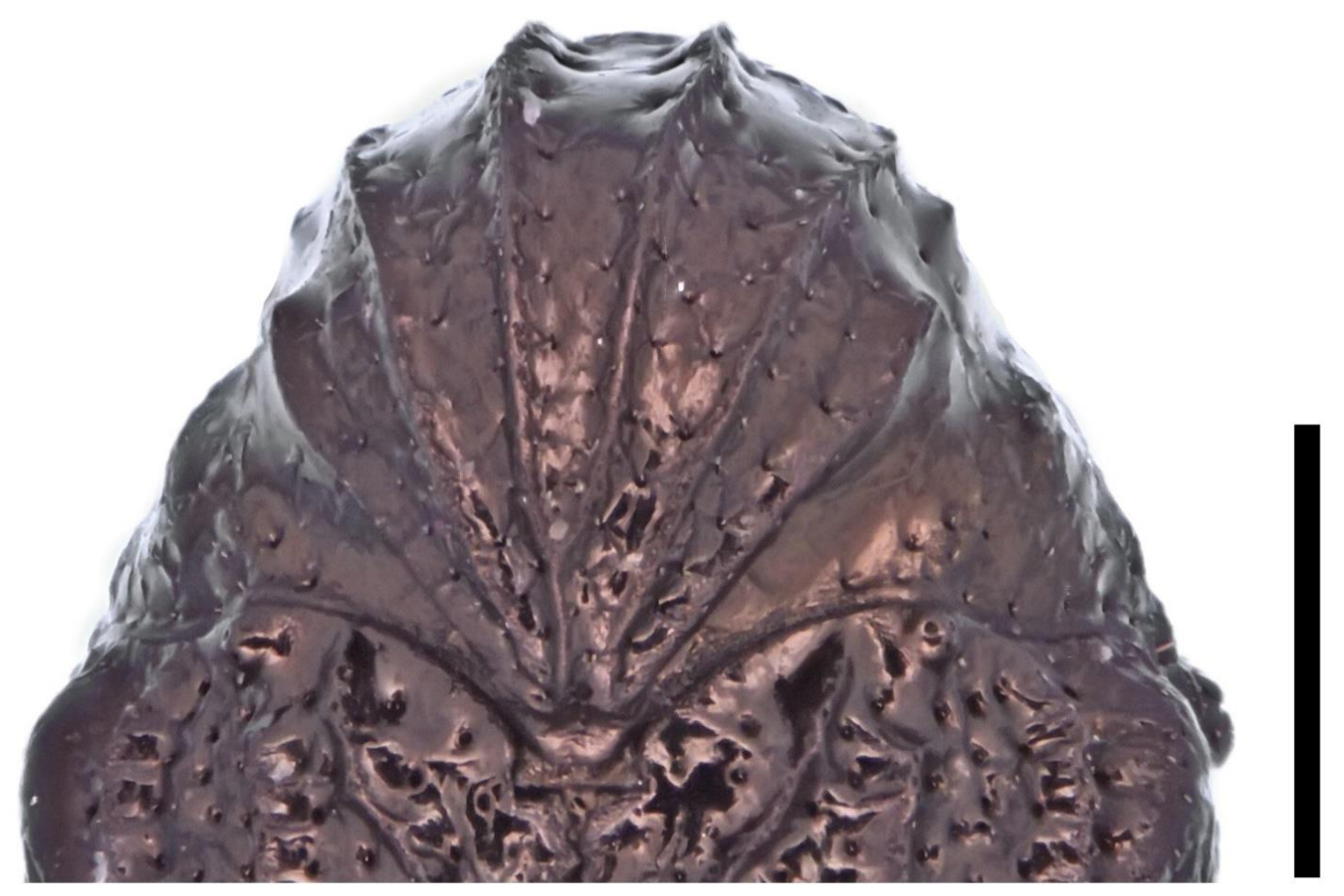

Figura 2. Aulacochlamys sp.; a) vista dorsal del pronoto (Escala de la barra $500 \mu \mathrm{m}$ ).

\section{Lista de especies mexicanas \\ 1. Aulacochlamys punctatipes (Lacordaire, 1848), nuevo estatus.}

\section{Género Chlamisus Rafinesque, 1815}

Es el primer género que se describió para la tribu, su nombre original fue Chlamys Knoch, 1801, que al resultar homónimo con Chlamys Bolten (1798) un género de molusco, se invalidó de acuerdo con el Código Internacional de Nomenclatura Zoológica. Boloschesis Jakobson, 1924 es también un sinónimo que destaca porque en 1926 Schaeffer realizó uno de los trabajos más completos del grupo para ese entonces. Chlamisus es el género con mayor diversidad en tamaño, forma y color. En general la forma del cuerpo es cilíndrica, mide de $2.1-6.0 \mathrm{~mm}$, existen de color amarillo, café, azul, rojo, verde, entre otros, pueden ser brillantes, metálicos o mate a lo largo del cuerpo, algunas especies presentan una combinación de colores. En vista dorsal y lateral se observan los tubérculos pronotales y elitrales que pueden ser ligeros o muy desarrollados, la superficie es punteada glabra o pubescente, escutelo visible, el pronoto casi siempre muy convexo, es decir se eleva en la parte media, metaescutelo no expuesto y proesterno no cóncavo en la parte media. Antena con el segundo antenómero poco ensanchado, más allá del $3^{\circ} \mathrm{o} 4^{\circ}$, con forma aserrada y por lo general el quinto casi tan ancho como el sexto.

Este género suele confundirse con Neochlamisus o Exema, la diferencia con el primero consiste en la simetría de la guía eyaculatoria que forma parte del edeago; en Chlamisus es simétrica y en Neochlamisus es asimétrica. Con respecto al segundo, se distingue porque el macho de Chlamisus no presenta espinas en el primer segmento abdominal y el quinto antenómero es casi tan ancho como el sexto. En contraste los machos de Exema presentan espinas o espínulas en el primer ventrito y el quinto antenómero menor que el sexto. 


\section{Lista de especies mexicanas}

2. Chlamisus admirabilis (Jacoby, 1889) (Tamaulipas)

3. Chlamisus angulicollis (Jacoby, 1889) (Jalisco)

4. Chlamisus aureopilosus (Bowditch, 1913) (Baja California, Veracruz)

5. Chlamisus balyi (Jacoby, 1904)

6. Chlamisus bipunctata (Jacoby, 1880) (Durango)

7. *Chlamisus brunnea (Jacoby, 1889) (Guerrero) (CCFES-Z)

8. Chlamisus carbonaria (Lacordaire, 1848)

9. Chlamisus cinerea (Lacordaire, 1848) (Baja California, Guerrero)

10. Chlamisus clarki (Jacoby, 1881)

11. Chlamisus coelocephala (Lacordaire, 1848)

12. *Chlamisus collaris (Jacoby, 1889) (Baja California, Guerrero, Morelos, Puebla, Veracruz) (CCFES-Z, UAT)

13. Chlamisus crassa (Lacordaire, 1848)

14. Chlamisus decipiens (Lacordaire, 1848)

15. *Chlamisus episcopalis (Lacordaire, 1848) (Baja California, Guerrero, Morelos, Oaxaca, Tamaulipas Veracruz) (CCFES-Z, UAT)

16. Chlamisus facialis (Lacordaire, 1848) (Baja California, Veracruz)

17. Chlamisus ferrugata (Lacordaire, 1848) (Baja California)

18. Chlamisus flavidus Karren, 1972 (Sonora)

19. Chlamisus foveolata (Knoch, 1801)

20. Chlamisus frontalis (Jacoby, 1881) (Oaxaca)

21. Chlamisus fulvicollis (Jacoby, 1881) (Oaxaca)

22. Chlamisus fulvomaculata (Jacoby, 1889)

23. Chlamisus gaumeri (Jacoby, 1889)

24. Chlamisus gnatho (Lacordaire, 1848)

25. Chlamisus granulicollis (Lacordaire, 1848)

26. Chlamisus gysselini (Kollar, 1824)

27. Chlamisus hoegei (Jacoby, 1889)

28. Chlamisus huachucae (Schaeffer, 1906) (Baja California, Coahuila, Sonora, Veracruz)

29. Chlamisus hybrida (Kollar, 1824)

30. Chlamisus hypocrita (Lacordaire, 1848)

31. *Chlamisus insidiosa (Lacordaire, 1848) (Baja California, Guerrero, Jalisco, Morelos) (CCFES-Z, UAT)

32. Chlamisus instabilis (Jacoby, 1889) (Morelos)

33. Chlamisus insularis (Jacoby, 1881) (Baja California, Morelos)

34. Chlamisus kraatzi (Jacoby, 1881)

35. Chlamisus luteola (Germar, 1824)

36. Chlamisus maculifrons (Jacoby, 1889) (Baja California, Durango)

37. *Chlamisus maculipes (Chevrolat, 1835) (Baja California, Campeche, Colima, Chiapas, Guerrero, Hidalgo, Jalisco, Morelos, Nayarit, Oaxaca, Puebla, San Luis Potosí, Tabasco, Tamaulipas, Veracruz) (CNIN, CCFES-Z, UACh, UAT, CEAM, ENCB, COLPOS-T)

38. Chlamisus mexicana (Lacordaire, 1848)

39. *Chlamisus militaris (Jacoby, 1889) (primer registro para México) (Chiapas) (CEAM)

40. Chlamisus mixta (Lacordaire, 1848)

41. Chlamisus modesta (Lacordaire, 1848)

42. *Chlamisus pardalis (Lacordaire, 1848) (Baja California, Quintana Roo, Veracruz) (UAT)

43. Chlamisus pectoralis (Jacoby, 1889) (Baja California, Veracruz)

44. Chlamisus pilatei (Jacoby, 1881) (Baja California, Tamaulipas, Veracruz)

45. Chlamisus punctipennis (Jacoby, 1889) 
46. *Chlamisus quadrilobatus (Schaeffer, 1926) (Baja California, Campeche, Colima, Chiapas, Estado de México, Guerrero, Hidalgo, Jalisco, Michoacán, Morelos, Nayarit, Oaxaca, Puebla, San Luis Potosí, Sinaloa, Sonora, Tabasco, Tamaulipas, Veracruz) (UAT, CCFES-Z, CEAM, CEFS, CNIN)

47. Chlamisus sallaei (Jacoby, 1881) (Oaxaca)

48. Chlamisus scabiosa (Lacordaire, 1848)

49. Chlamisus scabrosa (Lacordaire, 1889) (Baja California, Guerrero)

50. Chlamisus semifulva (Jacoby, 1889)

51. Chlamisus sextuberculata (Jacoby, 1879)

52. Chlamisus signaticollis (Lacordaire, 1848)

53. *Chlamisus stictica (Lacordaire, 1848) (Veracruz, Morelos) (CNIN)

54. *Chlamisus stigmula (Lacordaire, 1848) (Guerrero) (CCFES-Z)

55. Chlamisus sublaevicollis (Jacoby, 1889) (Baja California, Veracruz)

56. Chlamisus sumichrastii (Bowditch, 1913)

57. Chlamisus texanus (Schaeffer, 1906) (Tamaulipas, Yucatán)

58. *Chlamisus venusta (Lacordaire, 1848) (Baja California, Oaxaca, Veracruz) (CNIN)
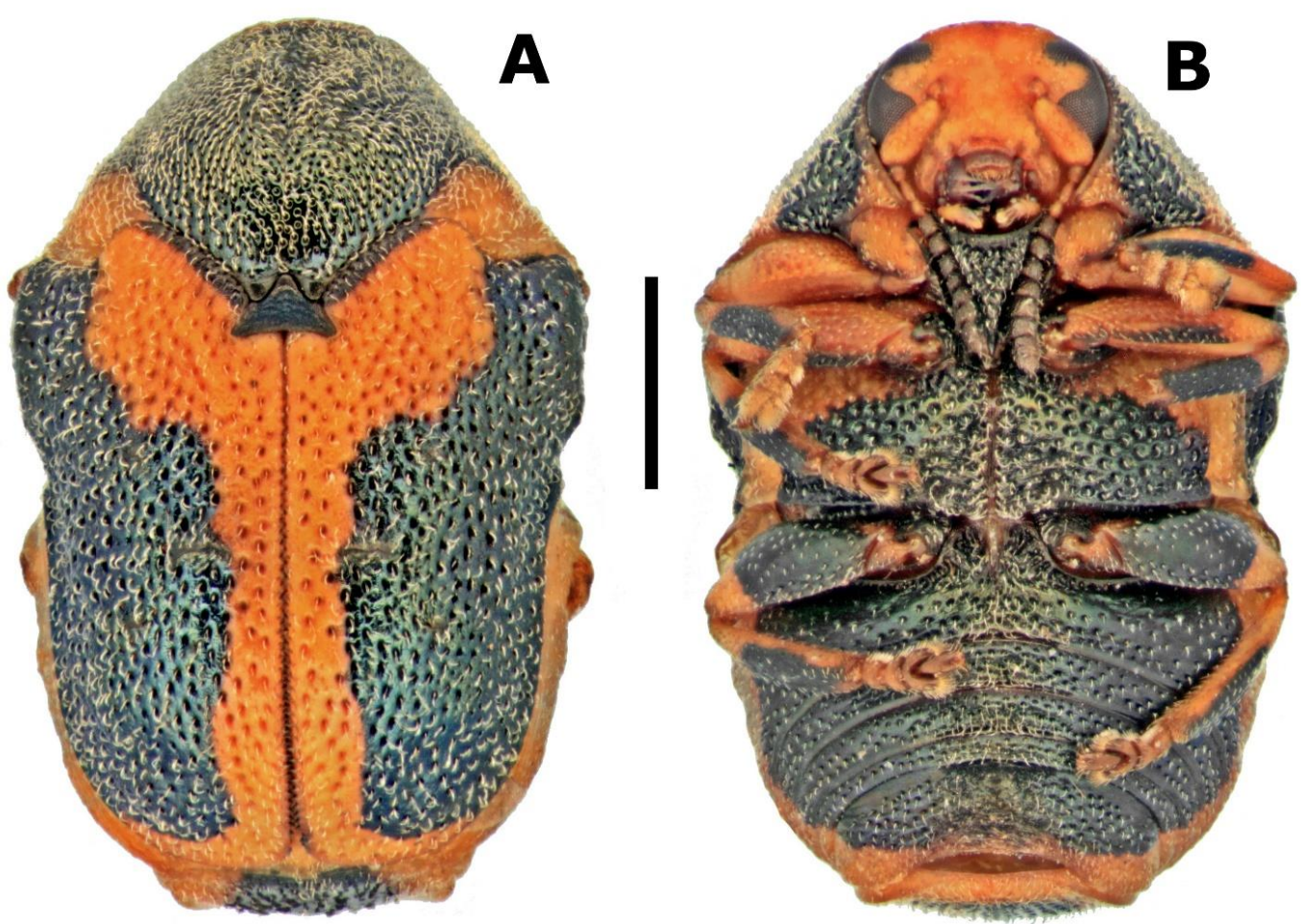

Figura 3. Chlamisus militaris (Jacoby, 1889); a) vista dorsal), b) vista ventral (Escala de la barra $1 \mathrm{~mm}$ ).

\section{Género Diplacaspis Jakobson, 1924}

Su primer nombre designado fue Diaspis, un género monotípico cuando fue descrito por Lacordaire (1848) al encontrar en D. paradoxa el metaescutelo visible y las uñas tarsales bífidas. En 1924 Jakobson propuso el nombre Diplacaspis para sustituir a Diaspis por ser homónimo de un género de la familia Coccidae (Hemiptera). Años posteriores, sin conocimiento de lo publicado por Jakobson, Emden (1932) propuso el nombre Skwarraia para sustituir a Diaspis, éste de nuevo resultó invalidado por la existencia de Diplacaspis. El género se caracteriza por presentar el metaescutelo expuesto, el proesterno cóncavo en la parte media, con ápice convexo y las uñas tarsales bífidas. En vista dorsal su cuerpo es claramente más ancho en la base 
de los élitros que en el ápice, son de color café-cobrizo, con numerosos tubérculos elitrales y pronotales, no muy grandes. Pronoto con dos elevaciones que se separan en la parte media por un surco, escutelo visible. Macho sin espinas o espínulas en el abdomen, edeago semejante al de Chlamisus.

Monrós (1951a) menciona que existen seis especies en el mundo sin especificar el nombre de las especies, para México se mantienen las dos especies ya registradas en la literatura.

\section{Lista de especies mexicanas}

59. Diplacaspis paradoxa (Lacordaire, 1848) (Baja California, Veracruz)

60. Diplacaspis prosternalis (Schaeffer, 1906) (Morelos)
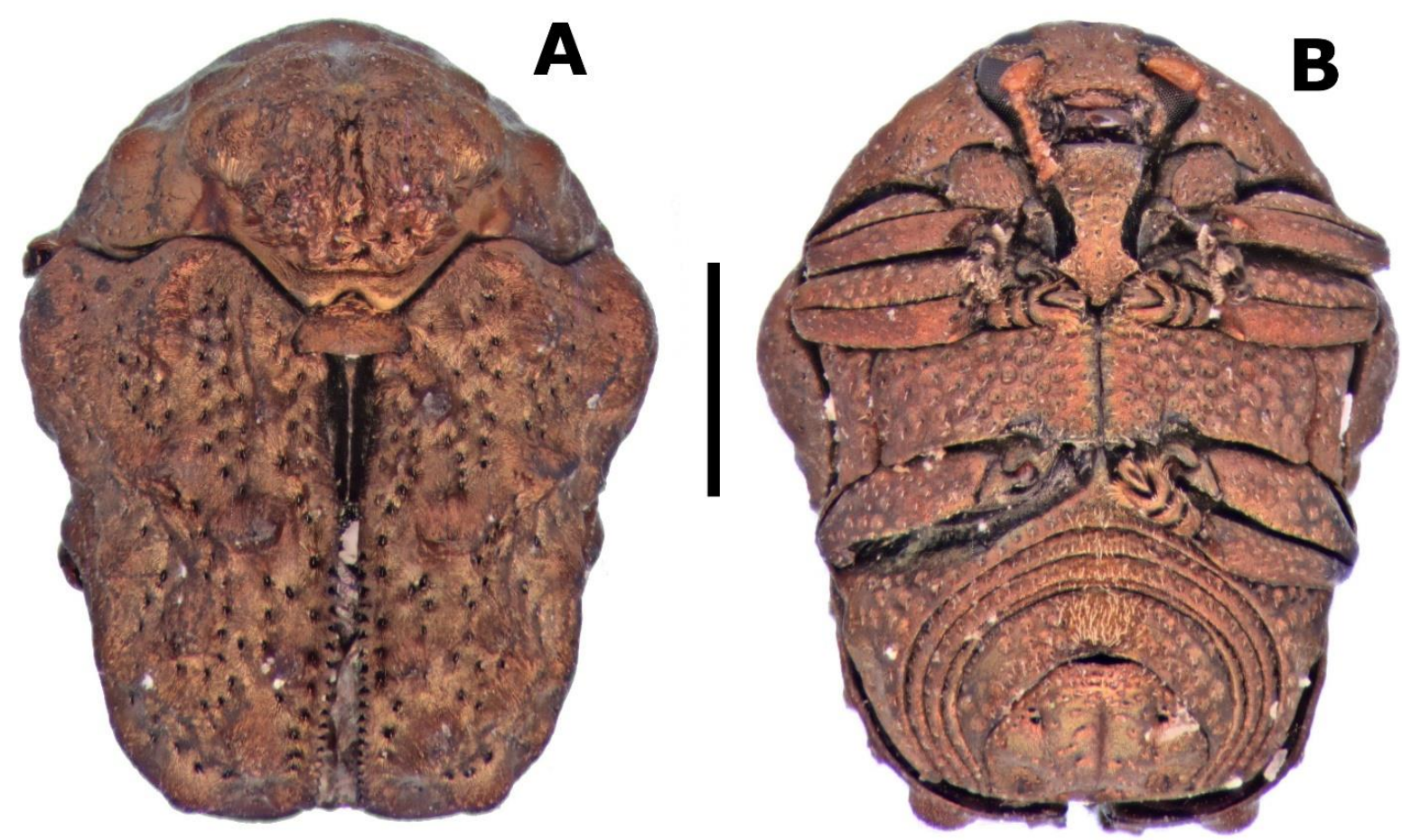

Figura 4. Diplacaspis sp.; a) vista dorsal), b) vista ventral (Escala de la barra $1 \mathrm{~mm}$ ).

\section{Género Exema Lacordaire, 1848}

Este género fue creado por Lacordaire (1848), al considerar que la forma antenal aserrada a partir del sexto segmento en conjunto con las uñas apendiculadas, era un carácter diferente al resto de fulcidacinos. El autor lo describe separándolo en dos divisiones; en la primera colocó a las especies que no tenían seis carinas pronotales y en el segundo las que sí presentan carinas. Antes de 1966 se dudó de la validez del género por su gran semejanza con Chlamisus, por ello Karren (1966) realizó una revisión del género, en donde afirmó que en todos los casos el quinto segmento antenal era menor que el sexto; esto aunado a la presencia de espinas o espinulas en el primer segmento abdominal del macho lo hicieron un género válido. De manera complementaria presentan un tamaño pequeño, cuerpo con forma cilíndrica, de color negro, café, mate o metálico, puede presentar manchas amarillas en desorden, proesterno más ancho en la base que se estrecha hacia el ápice. Edeago y espermateca semejantes a los de Chlamisus. 
De acuerdo con Monrós (1951a) en el mundo existen 26 especies y en la región Neotropical diez, para México se conocen cuatro especies.

\section{Lista de especies mexicanas}

61. *Exema canadensis Pierce, 1940 (Jalisco) (UAT, CEAM)

62. *Exema conspersa (Mannerheim, 1843) (Baja California Sur, Colima, Chiapas, Estado de México, Guanajuato, Guerrero, Hidalgo, Jalisco, Michoacán, Morelos, Nayarit, Oaxaca, Puebla, Querétaro, Quintana Roo, San Luis Potosí, Sonora, Tamaulipas) (CEAM, CCFES-Z, UAT, CEFS)

63. Exema deserti Pierce, 1940 (Baja California, Jalisco)

64. Exema dispar Lacordaire, 1848 (Chihuahua, Jalisco, Morelos, San Luis Potosí)
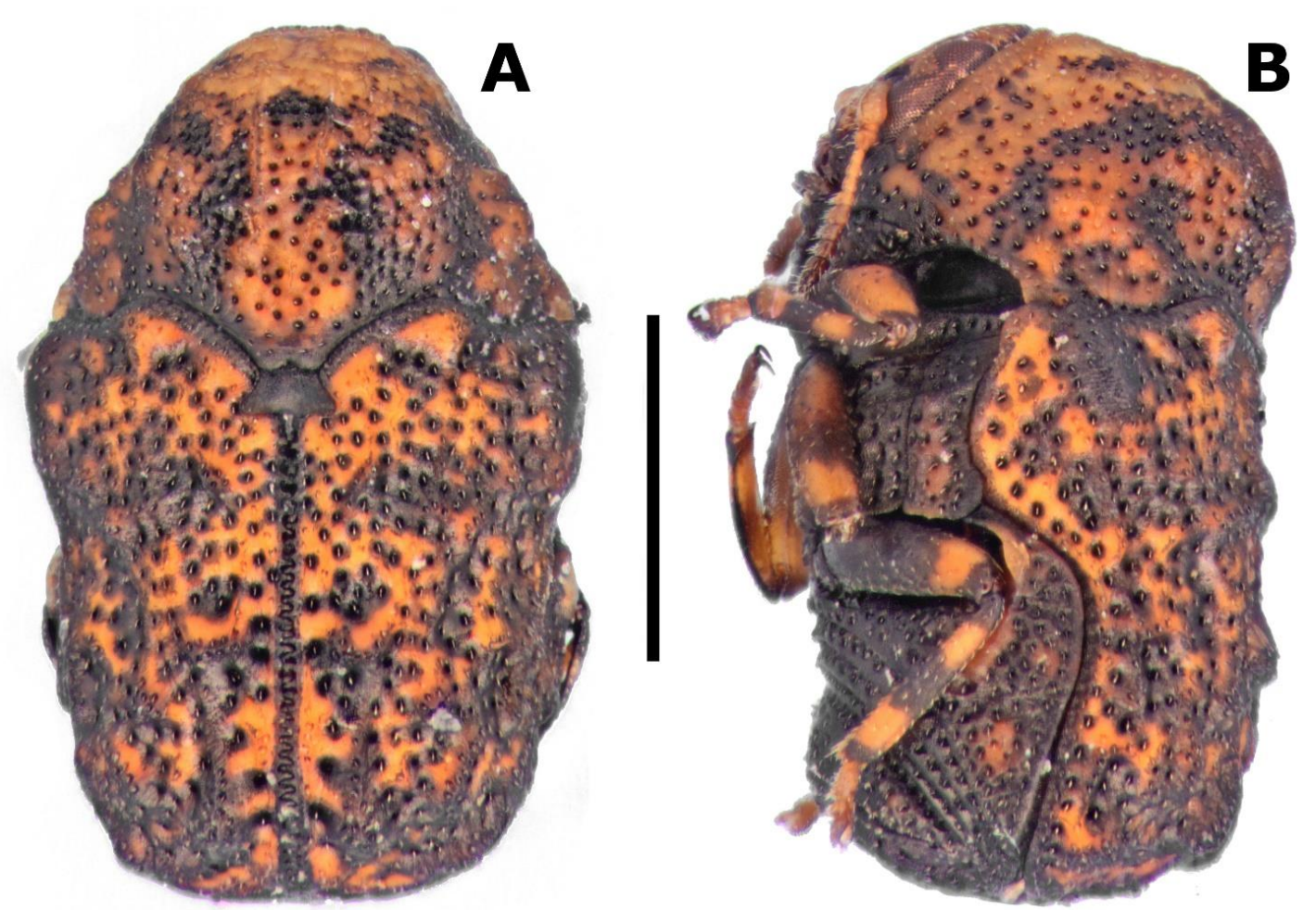

Figura 5. Exema conspersa (Mannerheim, 1843); a) vista dorsal), b) vista lateral (Escala de la barra $1 \mathrm{~mm}$ ).

\section{Género Fulcidax Voet, 1806}

El género fue creado como monotípico cuando Voet (1806) describió la primera especie Fulcidax azureus. Lacordaire (1848) en años siguientes, desconociendo dicho trabajo creó el género Poropleura con Poropleura monstrosa como especie tipo. Sin embargo, esta especie resultó una sinonimia de las especies Clytra monstrosa Fabricius, 1798 y Fulcidax azureus. Entonces por antiguiedad ya que Clytra es un género de la tribu Clytrini, el género Fulcidax se mantiene.

El género Fulcidax se distingue por ser el más vistoso, al ser el de mayor tamaño y tener los tubérculos más desarrollados de todo el grupo, además de presentar colores metálicos. Puede medir hasta $7.2 \mathrm{~mm}$ de longitud. Por su tamaño la diferencia entre macho y hembra es más evidente. En vista dorsal y lateral los tubérculos son grandes y numerosos, en la zona lateral del abdomen presentan un nódulo, las uñas tarsales son simples y cabeza con surco longitudinal en el vértex, las antenas son dentadas a partir del cuarto 
segmento. En machos el primer segmento abdominal nunca presenta espinas o espínulas, edeago muy semejante a Chlamisus con guía eyaculatoria simétrica.

De acuerdo con Monrós (1951a), en el mundo se conocen siete especies y en México sólo una especie se encuentra presente.

\section{Lista de especies mexicanas}

65. *Fulcidax bacca (Kirby, 1818) (Morelos, Tamaulipas) (UAT)
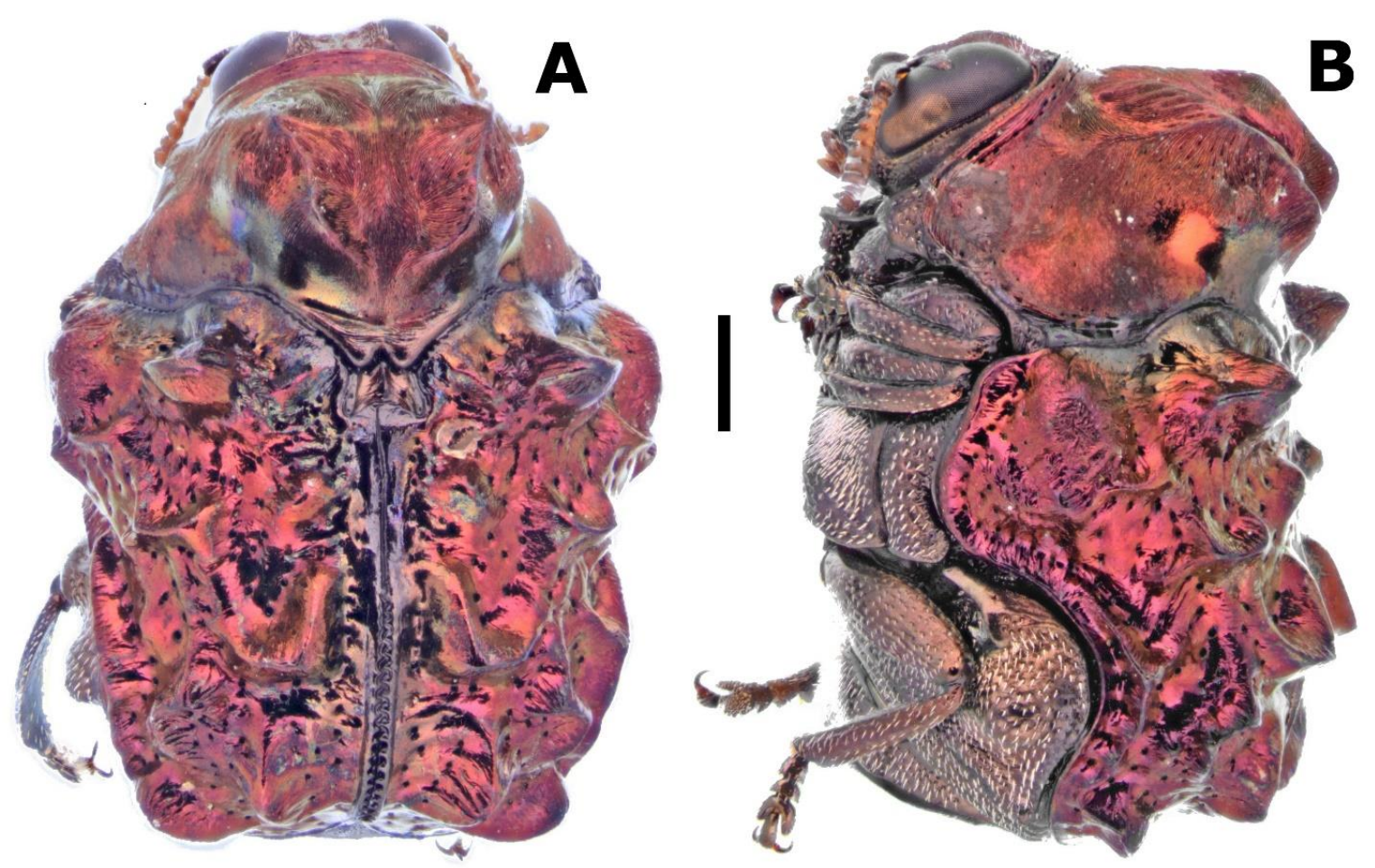

Figura 6. Fulcidax bacca (Kirby); a) vista dorsal), b) vista lateral (Escala de la barra $1 \mathrm{~mm}$ ).

\section{Género Melittochlamys Monrós, 1951}

Monrós (1951a) separó a este grupo del resto de los fulcidacinos, al observar que el proesterno tenía una forma rectangular alargada en el ápice y la presencia de depresiones en los élitros de apariencia aterciopelada.

Su forma es muy cercana a una circunferencia, de gran semejanza a la subfamilia Lamprosomatinae, de color café oscuro mate a negro tornasol por lo general, los tubérculos están poco desarrollados, el proesterno es rectangular truncado o algo escotado en el ápice, una característica que no comparte con otro género. En vista dorsal los élitros presentan una agrupación de puntos negros que vistos a distancia parecen puntos aterciopelados, élitros y pronoto están punteados, pueden ser glabros o pubescentes, de coloración café, negro o azulado. En machos el primer segmento abdominal nunca presenta espinas o espínulas; genitalia con la guía eyaculatoria simétrica. 


\section{Lista de especies mexicanas}

66. Melittochlamys amoena (Lacordaire, 1848) (Baja California, Veracruz)

68. Melittochlamys hydropica (Lacordaire, 1848)

67. *Melittochlamys pavonina (Lacordaire, 1848) (Baja California, Chiapas, Veracruz) (UAT)
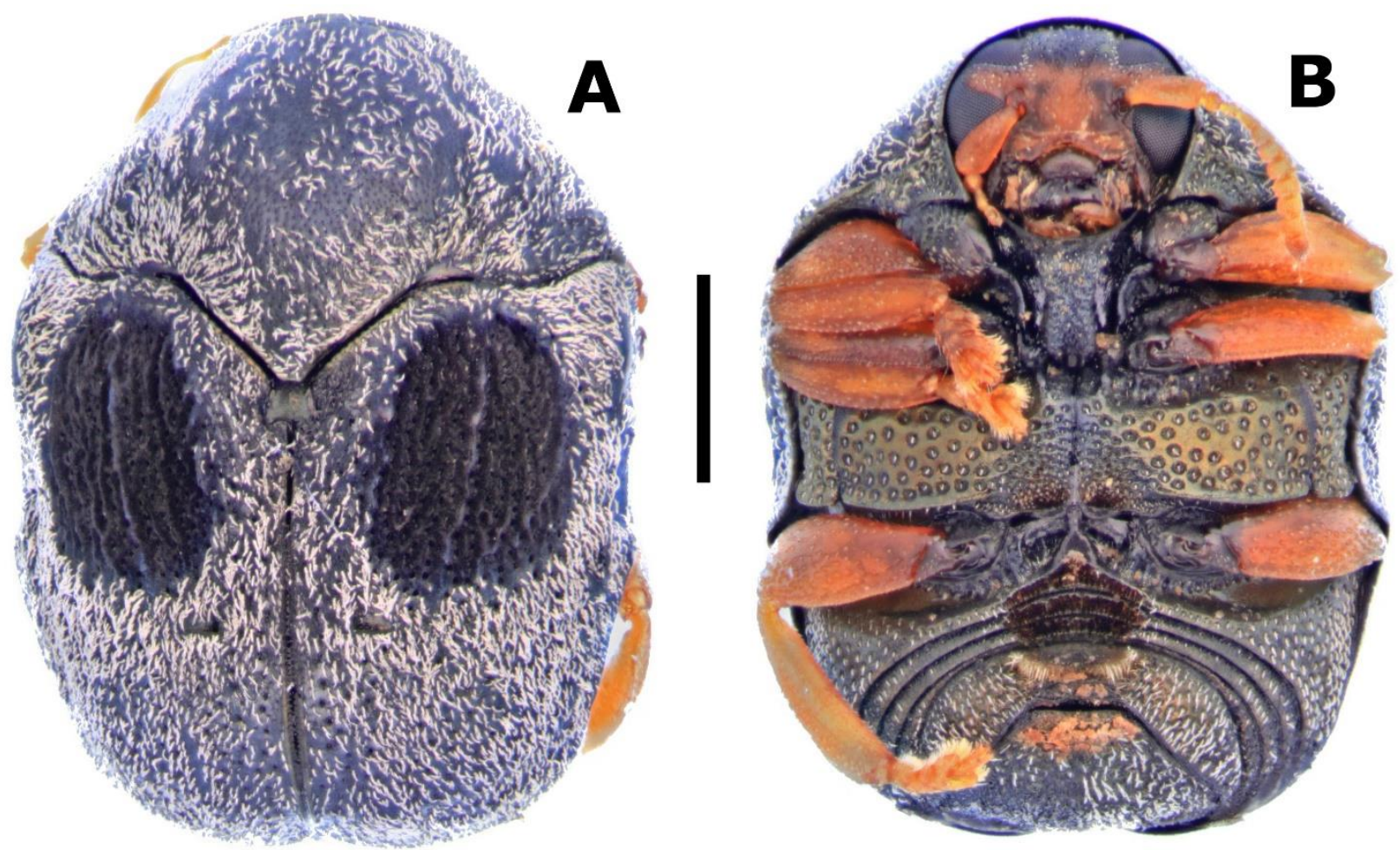

Figura 7. Melittochlamys pavonina (Lacordaire, 1948); a) vista dorsal), b) vista ventral (Escala de la barra $1 \mathrm{~mm}$ ).

\section{Género Neochlamisus Karren, 1972}

Es el género más reciente dentro de la tribu, fue creado por Karren (1972) en una de las revisiones más completas de la tribu, donde se consideraron especies de Norteamérica y norte de México. El autor al comparar las genitalias de los machos encontró que la guía eyaculatoria del edeago era distinta en este grupo, comparada con el resto de los géneros. Fue así como especies que antes pertenecieron a Chlamisus y Diplacaspis fueron trasladadas a Neochlamisus.

En la descripción del género Karren (1972) tomó como carácter distintivo las motas amarillas en el rostro y un conjunto de puntos que forman manchas de apariencia aterciopelada en los élitros. Sin embargo, en ninguna de las especies revisadas se observaron las motas y la luz fue un factor importante para distinguir los puntos elitrales, por ello para las especies mexicanas el carácter con mayor peso es la forma asimétrica de la guía.

La mayoría de las especies presentan colores cobrizos claro u oscuro con destellos metálicos o mate; pueden medir de 2.9 a $4.7 \mathrm{~mm}$ de longitud; cuerpo más ancho en la base de los élitros, estrechándose hacia el ápice (Chamorro-Lacayo \& Konstantinov, 2009).

Las especies del grupo tienen una gran semejanza con los géneros Chlamisus, Exema y Diplacaspis; por ejemplo, con Chlamisus y Exema se puede confundir por el tamaño, coloración y forma del cuerpo, en 
tanto que algunas especies de Neochlamisus presentan el metaescutelo expuesto como en Diplacaspis, pero el proesterno no es cóncavo en la parte media. En general Chlamisus, Exema y Diplacaspis se pueden separar fácilmente debido a que la guía eyaculatoria de la genitalia es simétrica.

En la revisión de especies de Norte América y norte de México Karren (1972) describió 17 especies. A continuación, se indican las especies registradas para México.

\section{Lista de especies mexicanas}

69. Neochlamisus bimaculatus Karren, 1972 (Chihuahua, Hidalgo, Jalisco)

70. Neochlamisus eubati (Brown, 1943)

71. Neochlamisus gibbosus (Fabricius, 1777)

72. *Neochlamisus memnonius (Lacordaire, 1848) (Baja California, Durango, Guerrero, Oaxaca,

Veracruz, Yucatán, Campeche, Guerrero, Jalisco, Oaxaca, Puebla) (CNIN)

73. Neochlamisus moestificus (Lacordaire, 1848) (Baja California, Chihuahua, Durango, Guerrero,

Oaxaca, Puebla, Sonora, Veracruz)

74. Neochlamisus scabripennis (Schaeffer, 1926) (Baja California, Chihuahua, Morelos, San Luis Potosí,

Veracruz, Zacatecas)

75. Neochlamisus subelatus (Schaeffer, 1926) (Chihuahua, Jalisco, Sonora)

76. *Neochlamisus velutinus Karren, 1972 (Baja California Sur, Coahuila, Chihuahua, Guerrero, Morelos, Nayarit, Nuevo León, San Luis Potosí, Tamaulipas) (CCFES-Z)
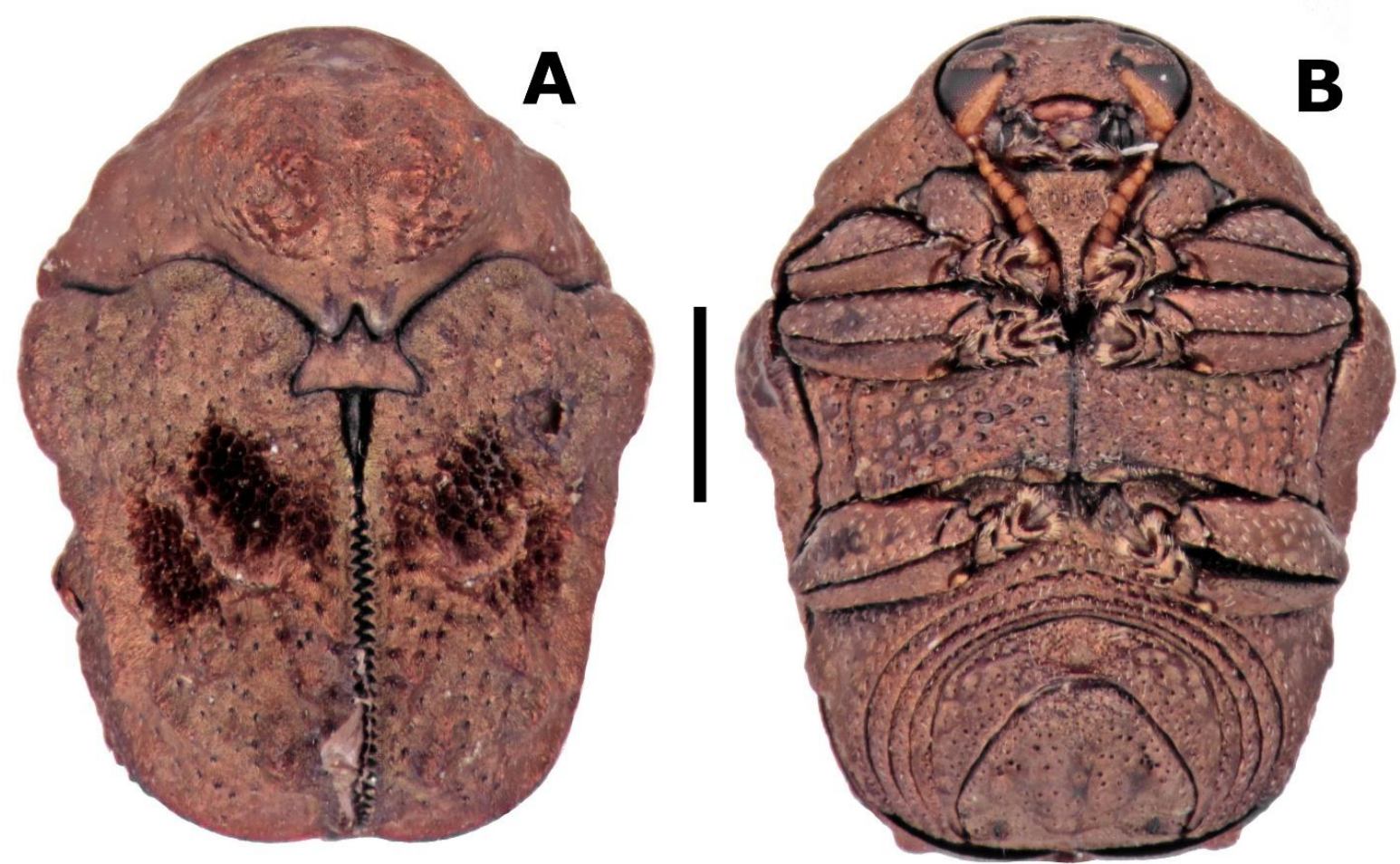

Figura 8. Neochlamisus memnonius (Lacordaire, 1848); a) vista dorsal, b) vista ventral (Escala de la barra 1mm). 


\section{DISCUSIÓN}

De la información contenida en literatura especializada y de los 341 ejemplares obtenidos de las colecciones nacionales en este estudio y el traslado genérico de una especie, en total se obtuvieron 76 especies de la tribu Fulcidacini para México, un número muy cercano a lo ya conocido en listados previos donde se habían reportado de 54 a 74 especies (Blackwelder, 1946; Ordóñez-Reséndiz, 2014). Al comparar la información contenida en el Catálogo de Autoridades Taxonómicas que realizó Ordóñez-Reséndiz (2014) se pudo observar que la mayoría de las especies son las mismas que se reportan para este estudio, es probable que este resultado se deba a que en dicho trabajo se incluyeron las especies registradas en literatura y especies que se localizaron en las colecciones entomológicas del National Museum of Natural History (NMNH, Smithsonian Institution); Museum of Comparative Zoology (MCZ, Harvard University); Centro Nacional de Referencia Fitosanitaria (SENASICA); Facultad de Estudios Superiores Zaragoza (UNAM); Instituto de Ecología, A.C. (INECOL) y en el Museo de Historia Natural de la Ciudad de México (MHNCA); sin embargo, por haberse elaborado sólo un listado no se pudo cotejar el destino de ese material, ya que colecciones como la de SENASICA y MHNCA no registraron material de la tribu, mientras que el MCZ, NMNH y INECOL no fueron revisadas. En este trabajo se registraron dos especies más con respecto al catálogo, una de ellas es Chlamisus brunnea (Jacoby, 1889) para el estado de Guerrero que OrdóñezReséndiz y López-Pérez (2009) publicaron como nuevo registro para el país y la otra fue Chlamisus militaris (Jacoby, 1889) que se registra por vez primera para México, ya que sólo había sido registrada en Guatemala. Además, se realizó el cambio genérico de Chlamisus memnonia (Lacordaire, 1848) a Neochlamisus memnonia (Lacordaire, 1848) y se cambió de género a Aulacochlamys punctatipes (Lacordaire, 1848) que antes estaba en el género Exema.

De acuerdo con el número de especies, México presenta un número alto comparado con lo reportado por Monrós (1951a) que obtuvo 47 especies y seis géneros presentes en Argentina después de revisar 30 colecciones (nacionales y extranjeras). A nivel nacional, de las ocho colecciones que fueron revisadas, el número de especies más alto se encontró en la UAT (nueve especies) y en CCFES-Z (seis especies) debido a que los curadores le han prestado especial atención a la captura de insectos que pertenecen a la familia Chrysomelidae.

En el mundo existen claves taxonómicas de las especies que habitan en Argentina (Monrós, 1951a), Norte América que incluyen algunas especies del norte de México (Karren, 1972) y China (Su \& Zhou, 2017). Sin embargo, la escasez de claves taxonómicas produce que el tiempo de identificación sea más lento, es por ello que 15 morfoespecies sólo fueron identificadas a nivel genérico, sin descartar que algunas podrían ser nuevas especies para la ciencia, por lo que es necesario continuar investigando a cerca de la tribu.

El cambio de E. punctatipes (Lacordaire, 1848) al género Aulacochlamys nos indica la escasez de estudios que se tienen del grupo a nivel taxonómico, porque pasaron muchos años sin modificación. Además, con las especies identificadas incluidas en los siete géneros se pudo observar qué tan claros eran los caracteres que definen a cada género. En la clave de los géneros de México se consideraron solo aquellos caracteres que no presentaban una alta variación como el tamaño de las especies del género Fulcidax y las motas amarillas en la frente que algunas claves mencionan para distinguir a Neochlamisus, haciendo de este modo más accesible la clave.

Agradecimientos. Al Consejo Nacional de Ciencia y Tecnología (CONACyT) por el financiamiento en la realización de esta investigación. A la Colección de Insectos del Colegio de Postgraduados, Montecillo, Estado de México (CEAM); a la Colección de Insectos del Colegio de Postgraduados, Tabasco (COLPOST); a la Universidad Autónoma Chapingo (UACh); a la Colección Nacional de Insectos del Instituto de Biología, UNAM (CNIN); a la Colección Coleopterológica de la Facultad de Estudios Superiores Zaragoza, 
UNAM (CCFES-Z); al Laboratorio de Entomología, Departamento de Zoología, Escuela Nacional de Ciencias Biológicas del Instituto Politécnico Nacional (ENCB); a la Universidad Autónoma de Tamaulipas (UAT) y El Colegio de la Frontera Sur, San Cristóbal de las Casas, Entomología (CEFS) por el préstamo del material para su revisión.

\section{LITERATURA CITADA}

Bezdĕk, J. (2010) Chrysomelidae: Cryptocephalinae: Fulcidacini. Pp: 76 In: Löbl, I., Smetana, A. (eds.) Catalogue of Palaearctic Coleoptera, Volume 6. Apollo Books, Stenstrup, Denmark.

Blackwelder, R. E. (1946) Checklist of the Coleopterous Insects of Mexico, Central America the West Indies, and South America. Bulletin of the United States National Museum, Smithsonian Institution, $185,(4), 647-650$.

Braga, S. R., Mesquita, A. L. M., Bandeira, C. T. (1999) Ocurrence of Fulcidax coelestina (Lac.) (Coleoptera: Chrysomelidae: Fulcidacinae) in Barbados Cherry Malpighia glabra L. Anais da Sociedade Entomologica do Brasil, 28 (3), 541-542.

Brown, W. J. (1952) Some species of Phytophaga (Coleoptera), Canadian Entomologist, 93, 967-977.

Brown, C. G., Funk, D. J. (2005) Aspects of the natural history of Neochlamisus (Coleoptera: Chrysomelidae): Fecal case-associated life history and behavior, with a method for studying insect constructions. Annals of the Entomological Society of America, 98, 711-725.

Bouchard, P., Bousquet Y., Davies, A. E., Alonso-Zarazaga, M. A., Lawrence, J. F., Lyal, C. H. C., Newton, A. F, Reid, C. A. M, Schmitt, M., Ślipiński, S. A., Smith, A. B. T. (2011) Family-group names in Coleoptera (Insecta). ZooKeys, 88, 1-972.

Chamorro-Lacayo, M. L., Konstantinov, A. S. (2009) Synopsis of warty leaf beetle genera of the World (Coleoptera, Chrysomelidae, Cryptocephalinae, Chlamisinae). ZooKeys, 8, 63-88.

Emden, F. Van. (1932) Die Larven von Dicoloma cassideum Teitt. Col. Colyd.) and Skwarraia paradoxa Lac. (Col. Chrysom. Zoologischers. Anzeiger, 101, 1-17.

Flowers, R. W., Hanson, P. E. (2003) Leaf beetle (Coleoptera: Chrysomelidae) diversity in eight Costa Rican habitats. Pp. 25-51. In: Furth, D. G. (ed.). Special Tropics in Leaf Beetle Biology. Proc. 5th Int. Sym. on the Chrysomelidae. PENSOFT publishers Sofia-Moscow.

Harley, K., Guillett, J., Winder, J., Forno, W., Segura, R., Miranda, H., Kassulke, R. (1995) Natural enemies of Mimosa pigra and M. berlandieri (Mimosaceae) and prospects for biological control of M. pigra. Environmental Entomology, 24 (6), 1664-1678.

Hyche, L. L. (1996) The sycamore leaf beetle. A guide to recognition and habits in Alabama. Alabama Agricultural Experiment Station. Bulletin 630, 12 p.

Jacoby, M. (1889) Biologia Centrali-Americana, Insecta. Coleoptera. Supplement Phytophaga (part) 6, 1, $155-172$.

Jolivet, P., Petitpierre E., Hsiao,T. H. (1988) Biology of Chrysomelidae. Kluwer Academic Publishers. Netherlands, $615 \mathrm{pp}$.

Jakobson, G. G. (1924) Annotationes synonymicae et systematieae de Coleopteris. Revue Russe d'Entomologie, 18, 237-243.

Karren, J. B. (1966) A revision of the genus Exema of America, north of Mexico (Chrysomelidae, Coleoptera). The University of Kansas Science Bulletin, 46 (18), 647-695.

Karren, J. B. (1972) A revision of the subfamily Chlamisinae of America north of Mexico (Coleoptera: Chrysomelidae). The University of Kansas Science Bulletin, 49, 875-988.

Karren, J. B. (1989) Chlamisus mimosae, n. sp., Coleoptera: Chrysomelidae: Chlamisinae) from Brazil and imported into Australia and Thailand. The Coleopterist Bulletin, 43 (4), 355-358.

Lacordaire, M. T. (1848) Monographie des Coléoptères subpentamères de la famille des Phytophages. Tome second. Bruxelles et Leipzig, 890 pp.

Monrós, F. (1951a) Revisión de las especies argentinas de Chlamisinae (Col., Chrysomelidae) (5a contribución al conocimiento de Chlamisinae). Acta Zoologica Lilloana, 10, 489-672. 
Monrós, F. (1951b) Notes on Chrysomelid beetles of the subfamily Chlamisinae, with descriptions of new species. Proceedings of the United States National Museum, 101, 451-463.

Ordóñez-Reséndiz, M. M. (2014) Catálogo de Autoridades Taxonómicas y base de datos curatorial de la familia Chrysomelidae en México. Universidad Nacional Autónoma de México. Facultad de Estudios Superiores Zaragoza. Informe final, SNIB-CONABIO. Proyecto No. HS003. México, D.F., 103 pp.

Ordóñez-Reséndiz, M. M., López-Pérez, S. (2009) Crisomélidos (Coleoptera: Chrysomelidae) de las Sierras de Taxco-Huautla, México. Entomología Mexicana, 8, 946-951.

Ostermeyer, N., Grace, B. S. (2006) Establishment, distribution and abundance of Mimosa pigra biological control agents in northern Australia: implications for biological control. BioControl, 52, 703-720.

Reid, C. A. M. (1991) The Australian species of Chlamisini (Coleoptera: Chrysomelidae). Journal of the Australian Entomological Society, 30, 315-323.

Schaeffer, C. F. A. (1926) New species of Boloschesis (=Chlamys) with notes on known species (Coleoptera; Chrysomelidae; Fulcidacinae). Proceeding Entomological Society Washington, 28, 181-187.

Su, L., Zhou, H-Z. (2017) Taxonomy of the genus Chlamisus Rafinesque (Coleoptera: Chrysomelidae) from China with description of three new species. Zootaxa, 4233 (1), 1-138.

Voet J. E. (1806) Catalogus systematicus coleopterorum, vol. 2. La Haye, 82 pp.

Waterhouse, D. F. (1994) Biological Control of Weeds: Southeast Asian Prospects. Australian Centre for International Agricultural Research (ACIAR), Monograph no. 26, 146-156.

Wood, G. W. (1966) Life History and Control of a casebearer, Chlamisus cribripennis (Coleoptera: Chrysomelidae), on blueberry. Journal of Economic Entomology, 59 (4), 823-825. 